\title{
IEEE 802.11 Packet Delay - A Finite Retry Limit Analysis
}

\author{
P. Chatzimisios, A. C. Boucouvalas and V.Vitsas* \\ Multimedia Communications Research Group, \\ School of DEC, Bournemouth University \\ Poole, UK \\ \{pchatzimisios, tboucouv\}@bournemouth.ac.uk, vitsas@it.teithe.gr
}

\begin{abstract}
The contribution of this paper is on the study of packet delays for the IEEE 802.11 wireless local area network DCF MAC protocol. A method is presented capable of taking into account retransmission delays with or without retry limits. We present an analytical model based on a Markov chain which allows us to derive closed form expressions for the packet delays, the probability of a packet being discarded when it reaches the maximum retransmission limit and the average time to drop such a packet for the basic and RTS/CTS access mechanisms. The results presented are for standard protocol parameters versus the number of contention stations. Finally, the accuracy of the analytical model is verified by simulations.
\end{abstract}

Keywords; wireless; IEEE 802.11; packet delay; retry limit;

\section{INTRODUCTION}

Wireless local area networking (WLAN) is a very dynamic field and a rapidly growing communication business. Technological and regulatory developments have allowed the issues of high prices, low data rates and licensing requirements to be addressed and the popularity of wireless LANs has grown significantly over the past few years. With wireless networking, regardless of where end users are within range, they can have network connectivity and they are a mouse-click away from key information and applications.

To deal with wireless user connectivity needs, various wireless communication standards have been developed [1][2]. The IEEE 802.11 protocols are a significant development; they are now a mature technology for WLANs and can offer high data rates through 802.11a and 802.11b standards, [3] [4]. The specifications are detailed and include both the Medium Access Control (MAC) and the Physical Layer (PHY). The MAC incorporates two medium access methods, Distributed Coordination Function (DCF) and Point Coordination Function (PCF). DCF is an asynchronous data transmission function, which is best suited to delay insensitive data. On the other hand, the optional Point Coordination Function (PCF) is utilized, when time-bounded services are required according to the communications needs.

Under DCF, data packets are transferred via two schemes. The default scheme is called the basic access mechanism, which transmits the data packet after deferring if the medium is busy. The 802.11 standard also provides an optional way of transmitting data packets, namely the Request To Send/Clear To

\footnotetext{
* Department of Information Technology,

Technological Educational Institution, Thessaloniki, Greece
}

Send (RTS/CTS) reservation based scheme. This scheme uses the small RTS/CTS packets to reserve the medium when large packets are transmitted in order to reduce the duration of a collision and to deal with the hidden terminal problem [5].

In the literature, several papers have studied the performance of the IEEE 802.11 protocol using analytical modes [6] [7] or by means of simulation [8] [9]. Moreover, much research effort is focused on improving the performance of the 802.11 MAC [10][11] and showed that performance depends on both throughput and delay considerations.

Our work introduces a performance analysis of both basic access and RTS/CTS access mechanisms. A throughput mathematical analysis has been developed [12] that does not consider packet dropping when the retry limit is reached. This throughput model is extended in [13] to include packet dropping. We have reported the packet delay in [14], using the Markov chain model of [12] which does not consider retransmission limit on collisions. In this paper we provide a new packet delay analysis, which extends the analysis in [14] to include the effect of retry limits. More specifically, this delay analysis allows us to calculate the packet delay, the packet drop probability and the packet drop time of the 802.11 protocol. Additionally, our model is compared with a model [12] that does not consider packet dropping which occurs when the number of packet transmission retries attains its limit. By including the packet retry limit, we consider that the model predicts the 802.11 packet delay in an accurate way.

Our model assumes that the network consists of $n$ contending stations transmitting in ideal conditions (no errors occur in the channel and no hidden stations exist). We also consider that every station has always a packet available for transmission (saturated conditions). The key assumption in our model is that the collision probability of a transmitted packet is constant and independent of the retransmissions that this packet has suffered in the past.

This paper is organized as follows: Section II introduces the DCF of the IEEE 802.11 MAC and focuses on the backoff procedure. Section III reviews the mathematical model. In Section IV, we extend the model and develop an analytical method to predict packet delay. Section $\mathrm{V}$ provides various analysis results. These results allow us to determine how the protocol performance is affected by the retry limit. Finally, section VI concludes the paper. 


\section{DISTRIBUTED COORDINATION FUNCTION (DCF)}

DCF is based on the Carrier Sense Multiple Access with Collision Avoidance (CSMA/CA) technique and adopts a slotted Binary Exponential Backoff (BEB) scheme to reduce collisions due to stations transmitting simultaneously. Each node with a packet to transmit first senses the medium to ascertain whether it is in use. If the medium is sensed to be idle for a time interval greater than the Distributed Inter-Frame Space (DIFS), the station proceeds with the packet transmission. If the medium is sensed busy, the station defers transmission and initializes its random backoff interval. This backoff timer is decremented when the medium is idle and is frozen when the medium is sensed busy. After a busy period the backoff resumes only after the medium has been idle for longer than DIFS.

Every station maintains a station short retry count (SSRC) as well as a long retry count (SLRC), both of which take an initial value of zero for every new packet. The short retry count indicates the maximum number of retransmission attempts of a RTS packet or of a data packet when RTS/CTS is not used. The long retry count indicates the maximum number of retransmission attempts of a data packet when RTS/CTS is used. When either of these limits is reached, retry attempts cease and the packet is discarded. We assume an error free channel, no hidden stations and packets are retransmitted only when they encounter collisions. As a result, the long retry limit is not used in our analysis.

The random number for the backoff timer is chosen in the interval $(0, C W-1)$, where $C W$ is the contention window size. The value of $C W$ depends on the number of failed transmissions of a packet. At the first transmission attempt $W=C W_{\text {min }}$, which is the minimum contention window. After each retransmission due to a collision, $C W$ is doubled up to a maximum value, $W_{m^{\prime}}=C W_{\max }=2^{m^{\prime}} \cdot C W_{\min }$, where $W_{m^{\prime}}$ is the largest contention window size. Once the $C W$ reaches $C W_{\max }$, it will remain at the value of $C W_{\max }$ until it is reset. Therefore, we have:

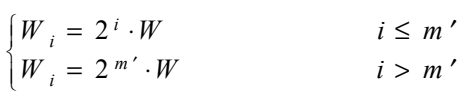

where $i$ is the backoff stage, $i \in[0, m]$ and $m$ represents the station short retry count. Here $m$ is also the maximum backoff stage. Our model considers that $m$ can have a value larger or smaller than $m^{\prime}$. The $802.11 \mathrm{~b}$ standard [4] specifies that $m=6$ and $m^{\prime}=5$.

After the successful reception of a packet in the destination station, an immediate positive acknowledgment (ACK) is sent back after a time interval equal to Short Inter-Frame Space (SIFS). Since SIFS is shorter than DIFS, the station sending an ACK attempts transmission before stations attempting to send new packets and hence takes priority. If the source station does not receive an ACK, the data packet is assumed to have been lost and a retransmission is scheduled.

\section{ANALYTICAL MODEL}

Let $b(t)$ and $s(t)$ be the stochastic processes representing the backoff timer and the backoff stage respectively for a given station at slot time $t$.

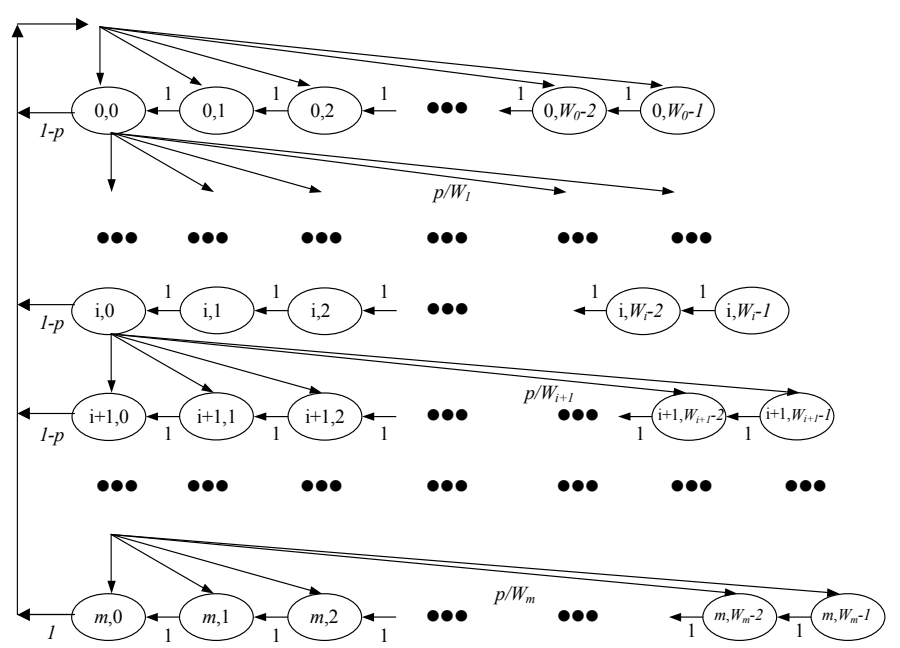

Fig. 1 Markov chain model

A discrete-time Markov chain showed in fig. 1 is used to model the bi-dimensional process $\{b(t), s(t)\}$. Let $b_{i, k}=\lim _{t \rightarrow \infty} P\{s(t)=i, b(t)=k\}$ be the stationary distribution of the Markov chain, where $i \in[0, m], k \in\left[0, W_{i}-1\right]$. We have the following relations:

$$
\begin{aligned}
& b_{i, 0}=p \cdot b_{i-1,0}, \quad 0<i \leq m \\
& b_{i, 0}=p^{i} \cdot b_{0,0} \quad, \quad 0<i \leq m
\end{aligned}
$$

Owing to the chain regularities and using (2), we have:

$$
b_{i, k}=\frac{W_{i}-k}{W_{i}} \cdot b_{i, 0}, i \in[0, m], k \in\left[0, W_{i}-1\right]
$$

Equations (3) and (4) express all $b_{i, k}$ values as a function of $b_{0,0}$ and $p$. If the normalization condition is imposed, finally $b_{0,0}$ is given by (5) and depends on the values of $m$ and $m^{\prime}$. Using the previous analysis, we can derive the probability $\tau$ that a station transmits a packet in a randomly chosen slot time. Since a station transmits when its backoff timer reaches the value of zero, $\tau$ can be found as:

$$
\tau=\sum_{i=0}^{m} b_{i, 0}=\sum_{i=0}^{m} p^{i} \cdot b_{0,0}=b_{0,0} \cdot \frac{1-p^{m+1}}{(1-p)}
$$

From (6) we can see that the transmission probability $\tau$ depends on the collision probability $p$, which is derived next.

$$
b_{0,0}= \begin{cases}\frac{2 \cdot(1-2 p) \cdot(1-p)}{W \cdot\left(1-(2 p)^{m+1}\right) \cdot(1-p)+(1-2 p) \cdot\left(1-p^{m+1}\right)} & , m \leq m^{\prime} \\ \frac{2 \cdot(1-2 p) \cdot(1-p)}{W \cdot\left(1-(2 p)^{m^{\prime}+1}\right) \cdot(1-p)+(1-2 p) \cdot\left(1-p^{m+1}\right)+W \cdot 2^{m^{\prime}} \cdot p^{m^{\prime}+1} \cdot(1-2 p) \cdot\left(1-p^{m-m^{\prime}}\right)} & , m>m^{\prime}\end{cases}
$$


The probability $p$ that a transmitted packet encounters a collision is the probability that at least one of the $n-1$ remaining stations transmit in the same time slot. If all stations transmit with probability $\tau$, the collision probability $p$ is:

$$
p=1-(1-\tau)^{n-1}
$$

Equations (6) and (7) form a nonlinear system with two unknowns $\tau$ and $p$. This nonlinear system can be solved utilizing numerical methods and has a unique solution. Note that $p \in[0,1]$ and $\tau \in[0,1]$.

Let $P_{t r}$ be the probability that at least one station transmits a packet in the considered slot time. For a wireless LAN of $n$ contending stations, $P_{t r}$ is given by:

$$
P_{t r}=1-(1-\tau)^{n}
$$

The probability $P_{s}$ that an occurring packet transmission is successful is given by the probability that exactly one station transmits and the remaining $n-1$ stations defer transmission, conditioned on the fact that at least one station transmits:

$$
P_{s}=\frac{n \cdot \tau \cdot(1-\tau)^{n-1}}{1-(1-\tau)^{n}}
$$

Considering that a random slot is empty with probability $\left(1-P_{t r}\right)$, contains a successful transmission with probability $P_{t r} \cdot P_{S}$ and a collision with probability $P_{t r} \cdot\left(1-P_{S}\right)$, the saturation throughput $S$ is given by:

$$
S=\frac{P_{t r} \cdot P_{S} \cdot l}{\left(1-P_{t r}\right) \cdot \sigma+P_{t r} \cdot P_{S} \cdot T_{S}+P_{t r} \cdot\left(1-P_{S}\right) \cdot T_{C}}
$$

where $l$ is the length of the transmitted packet, $\sigma$ the duration of an empty slot time, $T_{s}$ and $T_{c}$ are the average times that the medium is sensed busy due a successful transmission or a collision respectively. The values of $T_{s}$ and $T_{c}$ depend on the channel access mechanism and in the case of basic access:

$\left\langle\begin{array}{l}T_{S}^{\text {bas }}=D I F S+H+l+S I F S+A C K \\ T^{\text {bas }}=D I F S+H+l+S I F S+A C K\end{array}\right.$

For the RTS/CTS access mechanism, it is:

$\left\langle T_{S}^{R T S}=D I F S+R T S+S I F S+C T S+S I F S+H+l+S I F S+A C K\right.$

$T_{C}^{R T S}=D I F S+R T S+S I F S+C T S$

where $H=M A C_{h d r}+P H Y_{h d r}$ is the packet header.

\section{PaCKET Delay ANALYSIS}

Our analytical model considers the following metrics for the delay performance of IEEE 802.11 protocol, taking into account the retry limits of a data packet transmission.

\section{A. Packet drop probability}

The packet drop probability is defined, as the probability that a packet is dropped when the retry limit is reached and it is equal to:

$$
p_{\text {drop }}=p^{m+1}
$$

since a packet is dropped if it encounters $m+1$ collisions.

\section{B. Average time to drop a packet}

A packet is dropped when it reaches the last backoff stage and experiences another collision. The average value that the station will select for its backoff in the $i$ stage, is $\left(W_{i}+1\right) / 2$, where $i \in[0, m]$. Let $E\left[T_{\text {drop }}\right]$ be the average number of slot times required for a packet to experience $m+1$ collisions in the $(0,1, \ldots m)$ stages:

$$
E\left[T_{\text {drop }}\right]=\sum_{i=0}^{m} \frac{W_{i}+1}{2}= \begin{cases}\frac{W \cdot\left(2^{m+1}-1\right)+(m+1)}{2} & , m \leq m^{\prime} \\ \frac{W \cdot\left(2^{m+1}-1\right)+W \cdot 2^{m} \cdot\left(m-m^{\prime}\right)+(m+1)}{2} & , m>m^{\prime}\end{cases}
$$

The average length of a slot time is:

$$
E[s l o t]=\left(1-P_{t r}\right) \cdot \sigma+P_{t r} \cdot P_{s} \cdot T_{s}+P_{t r} \cdot\left(1-P_{s}\right) \cdot T_{c}
$$

Finally, the average time to drop a packet is equal to:

$$
E\left[D_{\text {drop }}\right]=E\left[T_{\text {drop }}\right] \cdot E[\text { slot }]
$$

\section{Average packet delay}

The average delay for a successfully transmitted packet is defined to be the time interval from the time the packet is at the head of its MAC queue ready to be transmitted, until an acknowledgement for this packet is received. If a packet is dropped because it has reached the specified retry limit, the delay time for this packet will not be included into the calculation of the average delay.

The average packet delay $E[D]$, provided that this packet is not discarded, is given by:

$$
E[D]=E[X] \cdot E[\text { slot }]
$$

where $E[X]$ is the average number of slot times required for successfully transmitting a packet and is given by:

$$
E[X]=\sum_{i=0}^{m}\left[\frac{\left(p^{i}-p^{m+1}\right) \cdot \frac{W_{i}+1}{2}}{1-p^{m+1}}\right]
$$

where $\left(1-p^{m+1}\right)$ is the probability that the packet is not dropped and $\left(p^{i}-p^{m+1}\right) /\left(1-p^{m+1}\right)$ is the probability that a packet that is not dropped reaches the $i$ stage. After some algebra, (16) becomes equal to (17).

$$
E[X]= \begin{cases}\frac{W \cdot\left(1-(2 p)^{m+1}\right) \cdot(1-p)+(1-2 p) \cdot\left(1-p^{m+1}\right)}{2 \cdot(1-2 p) \cdot(1-p)}-p^{m+1} \cdot E[\text { Tdrop }] & , m \leq m^{\prime} \\ \frac{W \cdot\left(1-(2 p)^{m^{\prime}+1}\right) \cdot(1-p)+W \cdot 2^{m^{\prime}} \cdot p^{m^{\prime}+1} \cdot\left(1-p^{m-m^{\prime}}\right) \cdot(1-2 p)+(1-2 p) \cdot\left(1-p^{m+1}\right)}{2 \cdot(1-2 p) \cdot(1-p)}-p^{m+1} \cdot E[\text { Tdrop }] & , m>m^{\prime}\end{cases}
$$




\section{ANALYSIS RESULTS}

Unless otherwise specified, the values reported in the following figures have been obtained using the system parameters in table I and are based on the Direct Spread Sequence Spectrum (DSSS) physical layer used in $802.11 \mathrm{~b}$ standard.

TABLE I DSSS SYSTEMS PARAMETERS IN 802.11b

\begin{tabular}{|l|l|}
\hline \multicolumn{1}{|c|}{ Parameter } & \multicolumn{1}{c|}{ Value } \\
\hline Packet payload, $l$ & 8184 bits \\
\hline Slot time, $\sigma$ & 20 us \\
\hline MAC header & 224 bits \\
\hline PHY header & 192 bits \\
\hline RTS packet & 160 bits + PHY header \\
\hline ACK packet & 112 bits + PHY header \\
\hline CTS packet & 112 bits + PHY header \\
\hline DIFS & $50 \mu \mathrm{s}$ \\
\hline SIFS & $10 \mu \mathrm{s}$ \\
\hline Channel bit rate & $1 \mathrm{Mbps}$ \\
\hline Minimum CW, $W_{0}$ & 32 \\
\hline Number of CW sizes, $m^{\prime}$ & 5 \\
\hline Short retry limit & 6 \\
\hline
\end{tabular}

Fig. 2 plots the packet delay against the number of stations and validates our analytical model since an almost exact match between analytical and simulation results is observed. Moreover, this figure reports the different packet delay values obtained from our model compared to results from a model that does not use any retry limits [12]. In both the cases of basic access and RTS/CTS mechanisms, the packet delay attained is higher when there are no retry limits for a packet transmission. This is easily justified by noting that in the case of no retry limits, a packet will be transmitted continuously until its successful reception.

The dependency of the average packet delay on the number of stations and the retry limit is examined in both the cases of the basic access and RTS/CTS mechanisms respectively in fig. 3 and 4 . Note that in fig.3, the vertical axis for the throughput efficiency is different to that in fig. 4. Both figures show that the average packet delay is an increasing function of the number of stations. This occurs because (i) a station has to wait longer due to the successful transmissions of other stations and (ii) more contending stations increase the probability of collisions and the number of retransmissions. The RTS/CTS mechanism provides a lower delay value than the basic access due to the smaller length of the colliding packets.

Also, fig. 3 and 4 report throughput and packet delay values for two different packet retry limits and for both basic access and RTS/CTS mechanisms. Note that the IEEE 802.11 standard proposes the value 6 for the retry limit. Results show that the retry limit considerably affects the throughput performance of the 802.11 protocol. Both figures illustrate that the average packet delay increases as the retry limit increases. and that the packet delay decreases if a smaller retry limit than the proposed value is employed, in both the basic access and RTS/CTS mechanisms. Especially in the case of medium or large network size $(n>15)$ the packet delay attains a lower value but at the expense of more packets being dropped.

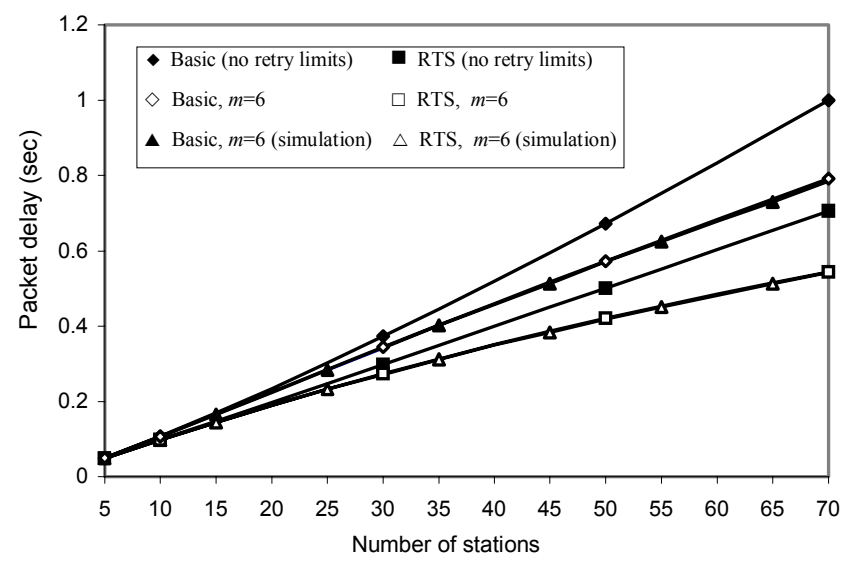

Fig. 2. Packet delay versus $\mathrm{n}$ for Basic access and RTS/CTS

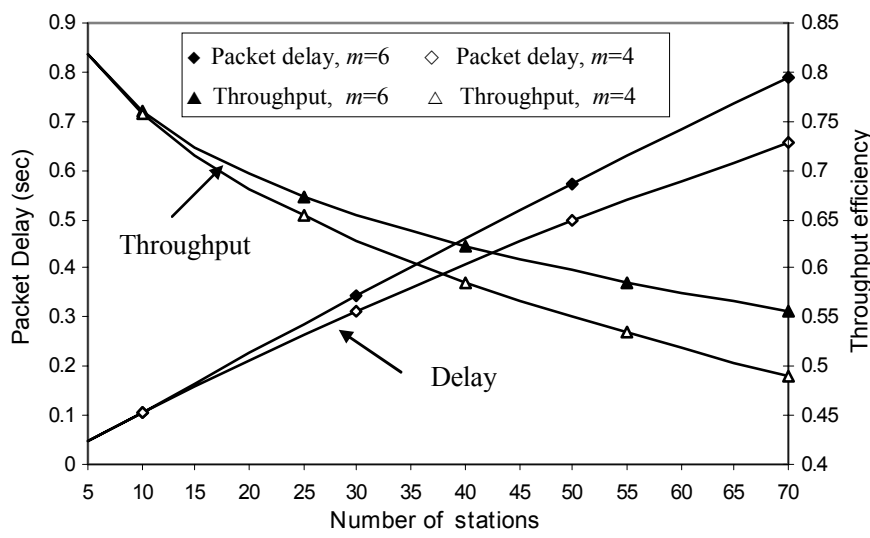

Fig. 3. Throughput efficiency and packet delay for basic access

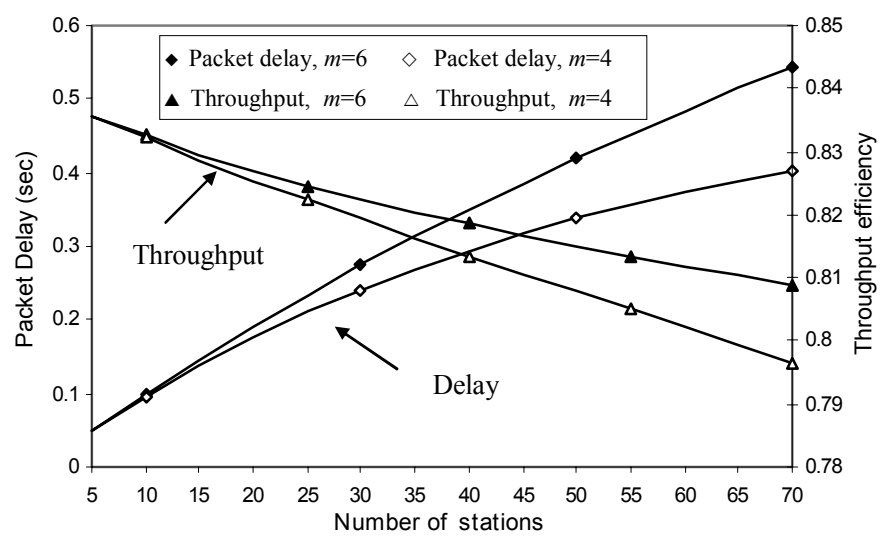

Fig. 4. Throughput efficiency and packet delay for RTS/CTS 


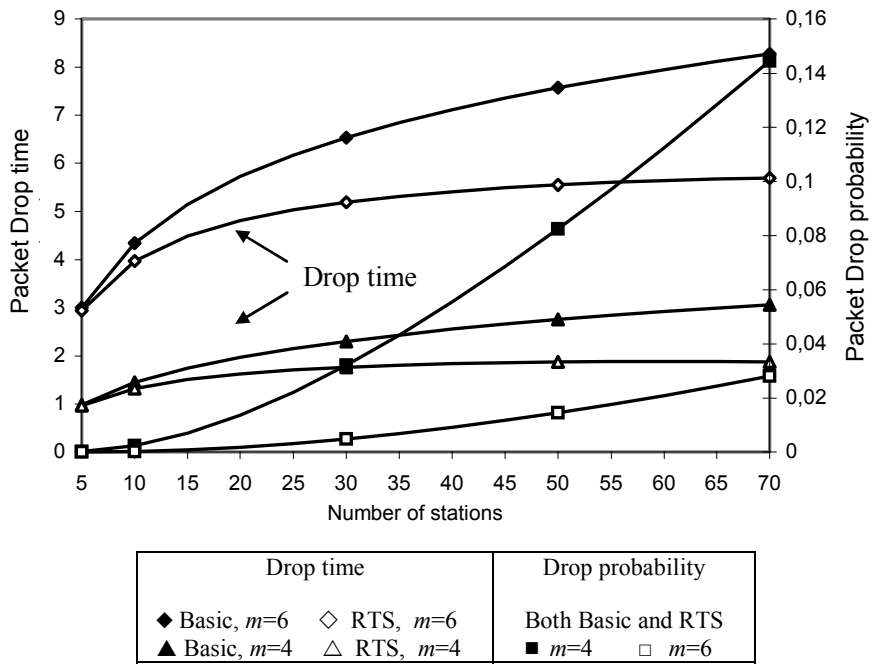

Fig. 5. Drop time and Drop probability versus $n$

Fig. 5 depicts the effects of the retry limit on the packet drop probability and the packet drop time. Some considerations are presented regarding the average drop time, namely the time in which a packet will be dropped because it reaches the maximum the retry limit. In all cases, the RTS/CTS mechanism achieves a lower value for the average drop time, with respect to the basic access mechanism. This decrease is mainly observable when the network size $n$ leads to a higher collision probability $(n=70)$. Furthermore, fig. 5 allows us to answer the question on the dependence of the average drop time on the retry limit. A small value of the retry limit $(m=4)$, results in a low average drop time. A higher value for the retry limit, $(m=6)$, corresponds to the higher average drop time of $8.4 \mathrm{~s}$ for $n=70$ if the basic access method is used.

Moreover, as shown in (11), the value of the packet drop probability depends on the retry limit and the collision probability. Since the packet drop probability does not depend on access mechanism, the results presented in fig. 4 are applicable on both basic access and RTS/CTS.

More specifically, when the retry limit is equal to the proposed value of the standard $(m=6)$, the packet drop probability increases as the number of stations increases. However, for small values of the retry limit and a large network size, the packet drop probability increases rapidly. As a result, a packet drop probability of 0.14 is obtained if the retry limit is $m=4$ for a network of 70 contending stations.

\section{CONCLUSIONS}

In this paper, an analytical model using a Markov chain was developed to compute the packet delay, the packet drop probability and the packet drop time protocol of IEEE 802.11 protocol. This analysis can be used in both the basic access and RTS/CTS methods and includes retry limits. Our proposed model, which takes into account packet retry limits, is compared to a model without any retry limits. Comparison with simulation results showed that our model can predict accurately the delay performance metrics of the 802.11 protocol. We also have shown that using packet retry limits results in a lower average delay for a packet transmission comparing to the case without retry limits, however, at the expense of finite packet drop probability.

\section{REFERENCES}

[1] IEEE standard for Wireless LAN Medium Access Control (MAC) and Physical Layer (PHY) specifications, ISO/IEC 8802-11:1999(E), Aug. 1999

[2] HiperLAN- High Performance Radio Local Area Network Draft ver. 1.1, ETSI, 1995.

[3] Wireless LAN Medium Access Control (MAC) and Physical Layer (PHY) Specification: High-Speed Physical Layer in the $5 \mathrm{GHz}$ Band, IEEE 802.11a WG, Sept. 1999.

[4] Wireless LAN Medium Access Control (MAC) and Physical Layer (PHY) Specification: High-Speed Physical Layer Extension in the $2.4 \mathrm{GHz}$ Band, IEEE 802.11b WG, Sept. 1999.

[5] K.Huang, K.Chen. "Interference Analysis of Nonpersistent CSMA with Hidden Terminals in Multicell Wireless Data Networks", Proc. PIMRC, Toronto, pp.907-911, 1995.

[6] H.S. Chhaya, S. Gupta, "Performance modeling of asynchronous data transfer methods of IEEE 802.11 MAC protocol", Wireless Networks, pp.217-234, 1997.

[7] Y. C. Tay , K. C. Chua, "A capacity analysis for the IEEE 802.11 MAC protocol", Wireless Networks, Volume 7, Issue 2, pp. 159171, March 2001.

[8] J. Weinmiller, H. Woesner, JP Ebert, A. Wolisz, "Analyzing and Tuning the Distributed Coordination Function in the IEEE 802.11 DFWMAC Draft Standard", Proc. of MASCOT'96, San Jose, California, 1996.

[9] Marek Natkaniec, Andrzej R. Pach, "Analysis of the Backoff mechanism in IEEE 802.11 standard".,IEEE Symposium on Computers and Communications, Antibes, France, July 2000.

[10] F.Cali, M.Conti, E.Gregori. "Dynamic Tuning of the IEEE 802.11 Protocol to Achieve a Theoretical Throughput Limit", IEEE/ACM Trans. On Networking, V8, N6, 2000.

[11] H. Wu, K. Long, S. Cheng, J. Ma, "IEEE 802.11 Distributed Coordination Function (DCF): Analysis and Enhancement", International Conference on Communications (ICC), April 2002.

[12] G.Bianchi. "Performance Analysis of the IEEE 802.11 Distributed Coordination Function", IEEE Journal on Selected Area in Comm. V18, N3, pp. 535-547, 2000.

[13] H. Wu, Y. Peng, K. Long, S. Cheng, J. Ma, "Performance of Reliable Transport Protocol over IEEE 802.11 Wireless LAN: Analysis And Enhancement", IEEE INFOCOM'2002.

[14] P.Chatzimisios, V.Vitsas, A. C. Boucouvalas, "Throughput and Delay analysis of IEEE 802.11 protocol" Proc. of IEEE International Workshop on Networked Appliances (IWNA), Liverpool, UK, pp. $168-174,2002$. 